\title{
Ratios of selected chemical element contents in prostatic tissue as markers of malignancy
}

\author{
Vladimir Zaichick ${ }^{1 *}$ and Sofia Zaichick ${ }^{2}$ \\ ${ }^{1}$ Radionuclide Diagnostics Department, Medical Radiological Research Centre, Koroleva Str-4, Obninsk-249036, Kaluga Region, Russia \\ ${ }^{2}$ Department of Medicine, University of Illinois College of Medicine, Chicago, IL 60612, USA
}

\begin{abstract}
The aim of the study was the development of new highly precise testing methods for early diagnosis of prostate cancer. For this purpose, the values of $\mathrm{Ca} / \mathrm{Al}$, $\mathrm{Ca} / \mathrm{B}, \mathrm{Ca} / \mathrm{Ba}, \mathrm{Ca} / \mathrm{Mn}, \mathrm{Mg} / \mathrm{Al}, \mathrm{Mg} / \mathrm{B}, \mathrm{Mg} / \mathrm{Ba}, \mathrm{Mg} / \mathrm{Mn}, \mathrm{S} / \mathrm{Al}, \mathrm{S} / \mathrm{B}, \mathrm{S} / \mathrm{Ba}, \mathrm{S} / \mathrm{Mn}, \mathrm{Zn} / \mathrm{Al}, \mathrm{Zn} / \mathrm{B}, \mathrm{Zn} / \mathrm{Ba}$, and $\mathrm{Zn} / \mathrm{Mn}$ mass fraction ratios in normal (n=37), benign hypertrophic $(\mathrm{n}=32)$ and cancerous $(\mathrm{n}=60)$ human prostate gland were investigated using a combination of non-destructive and destructive methods: instrumental neutron activation analysis and inductively coupled plasma atomic emission spectrometry, respectively. Mean values \pm standard error of mean $(\mathrm{M} \pm \mathrm{SEM}$ ) for mass fraction ratios in the normal tissue were as follows $\mathrm{Ca} / \mathrm{Al} 103 \pm 21, \mathrm{Ca} / \mathrm{B} 4320 \pm 805, \mathrm{Ca} / \mathrm{Ba} 2957 \pm 577, \mathrm{Ca} / \mathrm{Mn} 2061 \pm 325, \mathrm{Mg} / \mathrm{Al} 36.1 \pm 4.1, \mathrm{Mg} / \mathrm{B} 1599 \pm 239$, $\mathrm{Mg} / \mathrm{Ba} 1086 \pm 227, \mathrm{Mg} / \mathrm{Mn} 776 \pm 70$, S/Al $319 \pm$ 31, S/B $14749 \pm 2166$, S/Ba $9640 \pm$ 1968, S/Mn $6991 \pm 379, \mathrm{Zn} / \mathrm{Al} 41.3 \pm 9.7, \mathrm{Zn} / \mathrm{B} 1974 \pm 559, \mathrm{Zn} / \mathrm{Ba} 1003$ \pm 195 , and $\mathrm{Zn} / \mathrm{Mn} 875 \pm 214$, respectively. It was observed that in benign hypertrophic tissues the $\mathrm{Ca} / \mathrm{B}, \mathrm{Mg} / \mathrm{B}$, and $\mathrm{S} / \mathrm{B}$ mass fraction ratios are lower than normal levels while the $\mathrm{Mg} / \mathrm{Al}$ and $\mathrm{Mg} / \mathrm{Mn}$ ratio are significantly higher. In cancerous tissue the values of all ratios investigated were significantly lower than in normal and benign hypertrophic prostate. It was shown that the $\mathrm{Ca} / \mathrm{Ba}, \mathrm{Ca} / \mathrm{Mn}, \mathrm{Mg} / \mathrm{Al}, \mathrm{Mg} / \mathrm{Ba}, \mathrm{Zn} / \mathrm{Al}, \mathrm{Zn} / \mathrm{B}$, and $\mathrm{Zn} / \mathrm{Ba}$ mass fraction ratios are the most informative for a differential diagnosis among all investigated mass fraction ratios. Finally, we propose to use the $(\mathrm{Ca} / \mathrm{Ba}) \cdot(\mathrm{Mg} / \mathrm{Al}) \cdot(\mathrm{Zn} / \mathrm{Mn})$ and $(\mathrm{Ca} / \mathrm{Ba}) \cdot(\mathrm{Mg} / \mathrm{Al}) \cdot(\mathrm{S} / \mathrm{B}) \cdot(\mathrm{Zn} /$ $\mathrm{Mn}$ ) multiplications of mass fraction ratios in a needle-biopsy core as the most informative indicators for distinguishing malignant from benign prostate. Sensitivity, specificity, and accuracy of these tests were 100-9\%, 100-2\%, and 100-2\%, respectively. Further studies on larger number of samples are required to confirm our findings, to study the impact of the chemical element contents on prostate cancer etiology and to examine the long-term pathological outcome.
\end{abstract}

\section{Introduction}

The prostate gland may be a source of many health problems in men past middle age, the most common being benign prostatic hyperplasia $(\mathrm{BPH})$, and prostatic carcinoma (PCa). $\mathrm{BPH}$ is a noncancerous enlargement of the prostate gland leading to obstruction of the urethra and can significantly impair quality of life [1]. The prevalence of histological BPH is found in approximately $50-60 \%$ of males age 40 50 , in over $70 \%$ at 60 years old and in greater than $90 \%$ of men over $70[2,3]$. In many Western industrialized countries, including North America, PCa is the most frequently diagnosed form of noncutaneous malignancy in males and, except for lung cancer, is the leading cause of death from cancer [4-9]. Although the etiology of $\mathrm{BPH}$ and $\mathrm{PCa}$ is unknown, some electrolytes and trace elements have been highlighted in the literature in relation to the development of these prostate diseases [10-29].

Electrolytes and trace elements have essential physiological functions such as maintenance and regulation of cell function and signalling, gene regulation, activation or inhibition of enzymatic reactions, neurotransmission, and regulation of membrane function. Essential or toxic (mutagenic, carcinogenic) properties of chemical elements depend on tissue-specific need or tolerance, respectively [30]. Excessive accumulation, deficiency or an imbalance of the chemical elements may disturb the cell functions and may result in cellular degeneration, death, and malignant transformation [31].

In reported studies, significant changes of chemical element contents in hyperplastic and cancerous prostate in comparison with those in the normal prostatic tissue were observed [31-66]. Moreover, a significant informative value of $\mathrm{Zn}$ content as a tumor marker for PCa diagnostics was shown by us $[67,68]$. Hence it is possible that besides $\mathrm{Zn}$, some other chemical elements also can be used as tumor markers for distinguish between benign and malignant prostate.

Current methods applied for measurement of chemical element contents in samples of human tissue include a number of methods. Among these methods the instrumental neutron activation analysis with high resolution spectrometry of short-lived radionuclides (INAASLR) is a non-destructive and one of the most sensitive techniques. It allows measure the chemical element contents in a few milligrams tissue without any treatment of sample that avoids the possibility of changing the content of chemical elements during a sample preparation [69-72]. However the INAA-SLR allow only determine the mean mass fractions of 6-7 chemical elements in the tissue samples of normal and cancerous prostate glands $[15,21,28,65,66]$. The inductively coupled plasma atomic emission spectrometry (ICP-AES) is a more power analytical tool than INAA-SLR $[18,22,47]$ but sample digestion is

Correspondence to: Vladimir Zaichick, Radionuclide Diagnostics Department, Medical Radiological Research Centre, Koroleva Str-4, Obninsk-249036, Kaluga Region, Russia, Tel: +7 (48439) 60289; Fax: +7 (495) 956 1440; E-mail: vezai@obninsk.com

Key words: chemical elements; chemical elemental mass fraction ratios, prostate; benign prostatic hypertrophy; prostatic carcinoma; neutron activation analysis; inductively coupled plasma atomic emission spectrometry

Received: October 13, 2016; Accepted: November 23, 2016; Published: November 26, 2016 
a critical step in elemental analysis by this method. In our previous studies a combination of both analytical methods were offered for using when the results obtained for some chemical elements by ICPAES were under the control of INAA-SLR data [47].

Analytical studies of the $\mathrm{Al}, \mathrm{B}, \mathrm{Ba}, \mathrm{Br}, \mathrm{Ca}, \mathrm{Cu}, \mathrm{Fe}, \mathrm{K}, \mathrm{Li}, \mathrm{Mg}$, $\mathrm{Mn}, \mathrm{Na}, \mathrm{P}, \mathrm{S}, \mathrm{Si}, \mathrm{Sr}$, and $\mathrm{Zn}$ contents in normal, $\mathrm{BPH}$ and $\mathrm{PCa}$ tissue were done by us using the combination of INAA-SLR and ICP-AES methods, which allowed for the first time to obtain reliable results [22]. In particular, it was shown that in $\mathrm{PCa}$ the mean values of $\mathrm{Al}, \mathrm{B}, \mathrm{Ba}$, and $\mathrm{Mn}$ are higher while those of $\mathrm{Ca} \mathrm{Mg}, \mathrm{S}$, and $\mathrm{Zn}$ are lower than in healthy and hyperplastic prostates [22,73-78]. Obtained results formed the basis for a new method for differential diagnosis of $\mathrm{BPH}$ and $\mathrm{PCa}$, the essence of which was to determine the ratios of chemical element contents in the material of needle biopsy of prostate indurated site, because, in comparison with the absolute values of chemical element contents, using their content ratios is more suitable for diagnostics [79].

The present study had three aims. The main objective was to obtain reliable results about the chemical element contents and calculate the values of $\mathrm{Ca} / \mathrm{Al}, \mathrm{Ca} / \mathrm{B}, \mathrm{Ca} / \mathrm{Ba}, \mathrm{Ca} / \mathrm{Mn}, \mathrm{Mg} / \mathrm{Al}, \mathrm{Mg} / \mathrm{B}, \mathrm{Mg} / \mathrm{Ba}, \mathrm{Mg} /$ $\mathrm{Mn}, \mathrm{S} / \mathrm{Al}, \mathrm{S} / \mathrm{B}, \mathrm{S} / \mathrm{Ba}, \mathrm{S} / \mathrm{Mn}, \mathrm{Zn} / \mathrm{Al}, \mathrm{Zn} / \mathrm{B}, \mathrm{Zn} / \mathrm{Ba}$, and $\mathrm{Zn} / \mathrm{Mn}$ mass fraction ratios in intact prostate of healthy men aged over 40 years and in the prostate gland of age-matched patients, who had either BPH or PCa combining in consecutive order non-destructive INAA-SLR with destructive ICP-AES. The second aim was to compare the levels of chemical element ratios in normal, hyperplastic, and cancerous prostate, and the third aim was to evaluate the levels of chemical element ratios for diagnosis of prostate cancer.

All studies were approved by the Ethical Committees of the Medical Radiological Research Centre, Obninsk.

\section{Material and methods}

\section{Samples}

The patients studied $(n=92)$ were hospitalized in the Urological Department of the Medical Radiological Research Centre. All of them were European-Caucasian, citizens of Moscow and Obninsk (a small city in a non-industrial region $105 \mathrm{~km}$ south-west of Moscow). Transrectal puncture biopsy of suspicious indurated regions of the prostate was performed for every patient, to permit morphological study of prostatic tissue at these sites and to estimate their chemical element contents. In all cases the diagnosis has been confirmed by clinical and morphological results obtained during studies of biopsy and resected materials. The age of 32 patients with $\mathrm{BPH}$ ranged from 56 to 78 years, the mean being $66 \pm 6(\mathrm{M} \pm \mathrm{SD})$ years. The 60 patients aged 40-79 suffered from PCa (stage T1-T4). Their mean age was $65 \pm$ $10(\mathrm{M} \pm \mathrm{SD})$ years.

Intact (Norm) prostates were removed at necropsy from 37 men aged 41-87 who had died suddenly. All deceased were EuropeanCaucasian, citizens of Moscow. Their mean age was $55 \pm 11(\mathrm{M} \pm \mathrm{SD})$ years. The majority of deaths were due to trauma. Tissue samples were collected from the peripheral zone of dorsal and lateral lobes of their prostates, within 2 days of death and then the samples were divided into two portions. One was used for morphological study while the other was intended for chemical element analysis. A histological examination was used to control the age norm conformity, as well as to confirm the absence of microadenomatosis and latent cancer [15,21,28].

\section{Sample preparation}

All tissue samples were divided into two portions. One was used for morphological study while the other was intended for chemical element analysis. After the samples intended for chemical element analysis were weighed, they were freeze-dried and homogenized. The sample weighing about $10 \mathrm{mg}$ (for biopsy materials) and 50-100 mg (for resected materials) was used for chemical element measurement by INAA-SLR. The samples for INAA-SLR were sealed separately in thin polyethylene films washed beforehand with acetone and rectified alcohol. The sealed samples were placed in labelled polyethylene ampoules.

After NAA-SLR investigation the prostate samples were taken out from the polyethylene ampoules and used for ICP-AES. The samples were decomposed in autoclaves; $1.5 \mathrm{~mL}$ of concentrated $\mathrm{HNO}_{3}$ (nitric acid at $65 \%$, maximum (max) of $0.0000005 \% \mathrm{Hg}$; GR, ISO, Merck) and $0.3 \mathrm{~mL}$ of $\mathrm{H}_{2} \mathrm{O}_{2}$ (pure for analysis) were added to prostate tissue samples, placed in one-chamber autoclaves (Ancon-AT2, Ltd., Russia) and then heated for $3 \mathrm{~h}$ at $160-200^{\circ} \mathrm{C}$. After autoclaving, they were cooled to room temperature and solutions from the decomposed samples were diluted with deionized water (up to $20 \mathrm{~mL}$ ) and transferred to plastic measuring bottles. Simultaneously, the same procedure was performed in autoclaves without tissue samples (only $\mathrm{HNO}_{3}+\mathrm{H}_{2} \mathrm{O}_{2}+$ deionized water), and the resultant solutions were used as control samples.

\section{Instrumentation and methods}

Information detailing with the NAA-SLR and ICP-AES methods used and other details of the analysis was presented in our previous publication $[15,18,21,22,28,47,65,66]$.

\section{Certified reference materials}

For quality control, ten subsamples of the certified reference materials IAEA H-4 Animal muscle from the International Atomic Energy Agency (IAEA), and also five sub-samples INCT-SBF-4 Soya Bean Flour, INCT-TL-1 Tea Leaves and INCT-MPH-2 Mixed Polish Herbs from the Institute of Nuclear Chemistry and Technology (INCT, Warszawa, Poland) were analyzed simultaneously with the investigated prostate tissue samples. All samples of CRM were treated in the same way as the prostate tissue samples. Detailed results of this quality assurance program were presented in earlier publications $[18,22,47]$.

\section{Computer programs and statistic}

A dedicated computer program for INAA mode optimization was used [80]. All prostate samples for INAA-SLR were prepared in duplicate and mean values of chemical element contents were used in final calculation. For elements investigated by INAA-SLR and ICP-AES the mean of all results was used. Using the Microsoft Office Excel software, the summary of statistics, arithmetic mean, standard deviation, standard error of mean, minimum and maximum values, median, percentiles with 0.025 and 0.975 levels was calculated for $\mathrm{Ca} /$ $\mathrm{Al}, \mathrm{Ca} / \mathrm{B}, \mathrm{Ca} / \mathrm{Ba}, \mathrm{Ca} / \mathrm{Mn}, \mathrm{Mg} / \mathrm{Al}, \mathrm{Mg} / \mathrm{B}, \mathrm{Mg} / \mathrm{Ba}, \mathrm{Mg} / \mathrm{Mn}, \mathrm{S} / \mathrm{Al}, \mathrm{S} / \mathrm{B}$, $\mathrm{S} / \mathrm{Ba}, \mathrm{S} / \mathrm{Mn}, \mathrm{Zn} / \mathrm{Al}, \mathrm{Zn} / \mathrm{B}, \mathrm{Zn} / \mathrm{Ba}$, and $\mathrm{Zn} / \mathrm{Mn}$ mass fraction ratios in normal, benign hyperplastic and cancerous prostate tissue. The difference in the results between BPH and Norm, PCa and Norm, and PCA and BPH was evaluated by parametric Student's t-test and nonparametric Wilcoxon-Mann-Whitney $U$-test. For the construction of "individual data sets for ratios of chemical element mass fraction or multiplications of selected ratios in normal, benign hypertrophic and cancerous prostate" diagrams the Microsoft Office Excel software was also used. 


\section{Results}

Table 1 depicts certain statistical parameters (arithmetic mean, standard deviation, standard error of mean, minimal and maximal values, median, percentiles with 0.025 and 0.975 levels) of the $\mathrm{Ca} / \mathrm{Al}$, $\mathrm{Ca} / \mathrm{B}, \mathrm{Ca} / \mathrm{Ba}, \mathrm{Ca} / \mathrm{Mn}, \mathrm{Mg} / \mathrm{Al}, \mathrm{Mg} / \mathrm{B}, \mathrm{Mg} / \mathrm{Ba}, \mathrm{Mg} / \mathrm{Mn}, \mathrm{S} / \mathrm{Al}, \mathrm{S} / \mathrm{B}, \mathrm{S} / \mathrm{Ba}$, $\mathrm{S} / \mathrm{Mn}, \mathrm{Zn} / \mathrm{Al}, \mathrm{Zn} / \mathrm{B}, \mathrm{Zn} / \mathrm{Ba}$, and $\mathrm{Zn} / \mathrm{Mn}$ mass fraction ratios in normal, benign hypertrophic and cancerous prostate.

The ratios of means and the difference between mean values of
$\mathrm{Ca} / \mathrm{Al}, \mathrm{Ca} / \mathrm{B}, \mathrm{Ca} / \mathrm{Ba}, \mathrm{Ca} / \mathrm{Mn}, \mathrm{Mg} / \mathrm{Al}, \mathrm{Mg} / \mathrm{B}, \mathrm{Mg} / \mathrm{Ba}, \mathrm{Mg} / \mathrm{Mn}, \mathrm{S} / \mathrm{Al}, \mathrm{S} / \mathrm{B}$, $\mathrm{S} / \mathrm{Ba}, \mathrm{S} / \mathrm{Mn}, \mathrm{Zn} / \mathrm{Al}, \mathrm{Zn} / \mathrm{B}, \mathrm{Zn} / \mathrm{Ba}$, and $\mathrm{Zn} / \mathrm{Mn}$ mass fraction ratios in normal, benign hypertrophic and cancerous prostate are presented in Table 2.

Table 3 contains parameters of the importance (sensitivity, specificity and accuracy) of $\mathrm{Al}, \mathrm{B}, \mathrm{Ba}, \mathrm{Ca}, \mathrm{Mg}, \mathrm{Mn}, \mathrm{S}$, and $\mathrm{Zn}$ mass fraction for the diagnosis of PCa calculated in this work.

Figures 1-4 and 5 depict individual data sets for $\mathrm{Ca} / \mathrm{Al}, \mathrm{Ca} / \mathrm{B}$,

Table 1. Some statistical parameters of $\mathrm{Ca} / \mathrm{Al}, \mathrm{Ca} / \mathrm{B}, \mathrm{Ca} / \mathrm{Ba}, \mathrm{Ca} / \mathrm{Mn}, \mathrm{Mg} / \mathrm{Al}, \mathrm{Mg} / \mathrm{B}, \mathrm{Mg} / \mathrm{Ba}, \mathrm{Mg} / \mathrm{Mn}, \mathrm{S} / \mathrm{Al}, \mathrm{S} / \mathrm{B}, \mathrm{S} / \mathrm{Ba}, \mathrm{S} / \mathrm{Mn}, \mathrm{Zn} / \mathrm{Al}, \mathrm{Zn} / \mathrm{B}, \mathrm{Zn} / \mathrm{Ba}$, and Zn/Mn mass fraction ratios in normal, benign hyperplastic (BPH), and cancerous (PCa) prostate. M-arithmetic mean; SD-standard deviation; SEM-standard error of mean; Min-minimum value; Max-maximum value; Per. 0.025-percentile with 0.025 level; Per. 0.975-percentile with 0.975 level.

\begin{tabular}{|c|c|c|c|c|c|c|c|c|c|}
\hline Tissue & Ratio & Mean & SD & SEM & Min & Max & Median & Per.0.025 & Per.0.975 \\
\hline \multirow[t]{16}{*}{ Normal $n=25$} & $\mathrm{Ca} / \mathrm{Al}$ & 103 & 101 & 21 & 18.0 & 507 & 69.2 & 19.9 & 329 \\
\hline & $\mathrm{Ca} / \mathrm{B}$ & 4320 & 3778 & 805 & 421 & 16360 & 3127 & 447 & 12941 \\
\hline & $\mathrm{Ca} / \mathrm{Ba}$ & 2957 & 2705 & 577 & 503 & 10895 & 2119 & 620 & 9324 \\
\hline & $\mathrm{Ca} / \mathrm{Mn}$ & 2061 & 1557 & 325 & 787 & 7659 & 1669 & 808 & 6380 \\
\hline & $\mathrm{Mg} / \mathrm{Al}$ & 36.1 & 20.3 & 4.1 & 9.00 & 83.2 & 32.9 & 9.14 & 75.3 \\
\hline & $\mathrm{Mg} / \mathrm{B}$ & 1599 & 1147 & 239 & 262 & 3777 & 1340 & 293 & 3762 \\
\hline & $\mathrm{Mg} / \mathrm{Ba}$ & 1086 & 1090 & 227 & 213 & 4355 & 712 & 290 & 4213 \\
\hline & $\mathrm{Mg} / \mathrm{Mn}$ & 776 & 344 & 70 & 405 & 1638 & 681 & 417 & 1615 \\
\hline & $\mathrm{S} / \mathrm{Al}$ & 319 & 157 & 31 & 110 & 806 & 302 & 111 & 676 \\
\hline & $\mathrm{S} / \mathrm{B}$ & 14749 & 10389 & 2166 & 2882 & 31840 & 11739 & 2903 & 31608 \\
\hline & $\mathrm{S} / \mathrm{Ba}$ & 9640 & 9438 & 1968 & 2065 & 46175 & 6967 & 2694 & 33980 \\
\hline & $\mathrm{S} / \mathrm{Mn}$ & 6991 & 1857 & 379 & 3298 & 10203 & 7047 & 4164 & 10178 \\
\hline & $\mathrm{Zn} / \mathrm{Al}$ & 41.3 & 48.4 & 9.7 & 3.84 & 234 & 30.9 & 6.18 & 169 \\
\hline & $\mathrm{Zn} / \mathrm{B}$ & 1974 & 2682 & 559 & 220 & 12225 & 1174 & 258 & 8801 \\
\hline & $\mathrm{Zn} / \mathrm{Ba}$ & 1003 & 933 & 195 & 117 & 3918 & 581 & 191 & 3393 \\
\hline & $\mathrm{Zn} / \mathrm{Mn}$ & 875 & 1050 & 214 & 177 & 5335 & 585 & 203 & 3347 \\
\hline \multirow[t]{16}{*}{ BPH $n=11$} & $\mathrm{Ca} / \mathrm{Al}$ & 101 & 57 & 18 & 46.2 & 221 & 73.3 & 46.5 & 208 \\
\hline & $\mathrm{Ca} / \mathrm{B}$ & 1550 & 572 & 191 & 365 & 2302 & 1696 & 487 & 2221 \\
\hline & $\mathrm{Ca} / \mathrm{Ba}$ & 2034 & 832 & 251 & 796 & 3111 & 1915 & 823 & 3040 \\
\hline & $\mathrm{Ca} / \mathrm{Mn}$ & 1789 & 616 & 186 & 973 & 2982 & 1691 & 987 & 2829 \\
\hline & $\mathrm{Mg} / \mathrm{Al}$ & 58.8 & 28.4 & 9.0 & 27.2 & 127 & 51.2 & 29.3 & 117 \\
\hline & $\mathrm{Mg} / \mathrm{B}$ & 928 & 413 & 138 & 215 & 1453 & 971 & 282 & 1436 \\
\hline & $\mathrm{Mg} / \mathrm{Ba}$ & 1258 & 657 & 198 & 403 & 2421 & 1034 & 4792 & 2377 \\
\hline & $\mathrm{Mg} / \mathrm{Mn}$ & 1077 & 377 & 114 & 520 & 1710 & 1148 & 533 & 1665 \\
\hline & $\mathrm{S} / \mathrm{Al}$ & 431 & 214 & 68 & 227 & 939 & 345 & 237 & 854 \\
\hline & $\mathrm{S} / \mathrm{B}$ & 6694 & 3038 & 1013 & 2638 & 12977 & 6908 & 2820 & 12004 \\
\hline & $\mathrm{S} / \mathrm{Ba}$ & 9418 & 5273 & 1590 & 3487 & 21629 & 8361 & 3710 & 19680 \\
\hline & $\mathrm{S} / \mathrm{Mn}$ & 7735 & 1965 & 592 & 4507 & 11355 & 7276 & 4732 & 10913 \\
\hline & $\mathrm{Zn} / \mathrm{Al}$ & 59.0 & 31.0 & 9.8 & 12.9 & 109 & 53.4 & 16.3 & 107 \\
\hline & $\mathrm{Zn} / \mathrm{B}$ & 1360 & 1250 & 417 & 102 & 4432 & 1130 & 216 & 3902 \\
\hline & $\mathrm{Zn} / \mathrm{Ba}$ & 1373 & 674 & 203 & 534 & 2266 & 1251 & 534 & 2234 \\
\hline & $\mathrm{Zn} / \mathrm{Mn}$ & 1261 & 615 & 185 & 272 & 2462 & 1479 & 383 & 2292 \\
\hline \multirow[t]{16}{*}{$\operatorname{PCa} n=11$} & $\mathrm{Ca} / \mathrm{Al}$ & 5.24 & 6.51 & 1.96 & 0.982 & 19.3 & 1.63 & 1.03 & 17.7 \\
\hline & $\mathrm{Ca} / \mathrm{B}$ & 119 & 183 & 58 & 17.4 & 635 & 62.3 & 22.1 & 515 \\
\hline & $\mathrm{Ca} / \mathrm{Ba}$ & 102 & 157 & 47 & 8.27 & 422 & 38.6 & 8.80 & 420 \\
\hline & $\mathrm{Ca} / \mathrm{Mn}$ & 181 & 218 & 66 & 44.9 & 772 & 91.0 & 46.7 & 670 \\
\hline & $\mathrm{Mg} / \mathrm{Al}$ & 2.78 & 3.33 & 1.05 & 0.277 & 8.75 & 0.852 & 0.313 & 8.47 \\
\hline & $\mathrm{Mg} / \mathrm{B}$ & 70.1 & 132 & 44.2 & 8.35 & 421 & 31.6 & 9.08 & 346 \\
\hline & $\mathrm{Mg} / \mathrm{Ba}$ & 67.5 & 105 & 33.4 & 5.99 & 275 & 12.4 & 6.02 & 269 \\
\hline & $\mathrm{Mg} / \mathrm{Mn}$ & 107 & 133 & 42 & 9.07 & 458 & 74.1 & 10.2 & 389 \\
\hline & $\mathrm{S} / \mathrm{Al}$ & 37.0 & 39.9 & 12.0 & 7.52 & 119 & 15.2 & 7.66 & 112 \\
\hline & $\mathrm{S} / \mathrm{B}$ & 903 & 1298 & 410 & 167 & 4519 & 457 & 184 & 3752 \\
\hline & $\mathrm{S} / \mathrm{Ba}$ & 850 & 1324 & 399 & 84.3 & 3957 & 190 & 85.7 & 3704 \\
\hline & $\mathrm{S} / \mathrm{Mn}$ & 1476 & 1966 & 593 & 264 & 7241 & 1116 & 301 & 5834 \\
\hline & $\mathrm{Zn} / \mathrm{Al}$ & 1.16 & 1.73 & 0.52 & 0.119 & 4.53 & 0.231 & 0.126 & 4.48 \\
\hline & $\mathrm{Zn} / \mathrm{B}$ & 28.8 & 66.5 & 21.0 & 2.73 & 218 & 9.16 & 3.13 & 172 \\
\hline & $\mathrm{Zn} / \mathrm{Ba}$ & 29.5 & 56.5 & 17.0 & 1.26 & 145 & 3.51 & 1.28 & 144 \\
\hline & $\mathrm{Zn} / \mathrm{Mn}$ & 42.8 & 76.9 & 23.2 & 4.87 & 265 & 13.9 & 5.37 & 218 \\
\hline
\end{tabular}


Table 2. Ratio of means and the difference between mean values of $\mathrm{Ca} / \mathrm{Al}, \mathrm{Ca} / \mathrm{B}, \mathrm{Ca} / \mathrm{Ba}, \mathrm{Ca} / \mathrm{Mn}, \mathrm{Mg} / \mathrm{Al}, \mathrm{Mg} / \mathrm{B}, \mathrm{Mg} / \mathrm{Ba}, \mathrm{Mg} / \mathrm{Mn}, \mathrm{S} / \mathrm{Al}, \mathrm{S} / \mathrm{B}, \mathrm{S} / \mathrm{Ba}, \mathrm{S} / \mathrm{Mn}, \mathrm{Zn} / \mathrm{Al}, \mathrm{Zn} / \mathrm{B}, \mathrm{Zn} / \mathrm{Ba}$, and $\mathrm{Zn} / \mathrm{Mn}$ mass fraction ratios in normal, benign hypertrophic and cancerous prostate. $t$-test-Student's $t$-test, U-test-Wilcoxon-Mann-Whitney $U$-test, $B$ old significant differences.

\begin{tabular}{|c|c|c|c|c|c|c|c|c|c|}
\hline \multirow{2}{*}{$\begin{array}{l}\text { Mass fraction } \\
\text { ratio }\end{array}$} & \multicolumn{3}{|c|}{ BPH and Normal (N) } & \multicolumn{3}{|c|}{ PCa and Normal (N) } & \multicolumn{3}{|c|}{ PCa and BPH } \\
\hline & $\begin{array}{c}\text { Ratio } \\
\text { BPH/N }\end{array}$ & $\underset{t-\text { test }}{p}$ & $\begin{array}{c}p \\
\text { U-test }\end{array}$ & $\begin{array}{l}\text { Ratio } \\
\text { PCa/N }\end{array}$ & $\underset{t-\text { test }}{p}$ & $\begin{array}{c}p \\
\text { U-test }\end{array}$ & $\begin{array}{c}\text { Ratio } \\
\text { PCa/BPH }\end{array}$ & $\underset{t-\text { test }}{p}$ & $\begin{array}{c}p \\
\text { U-test }\end{array}$ \\
\hline $\mathrm{Ca} / \mathrm{Al}$ & 0.98 & $=0.94$ & $>0.05$ & 0.051 & $<0.00047$ & $\leq \mathbf{0 . 0 1}$ & 0.052 & $<0.00009$ & $\leq 0.01$ \\
\hline $\mathrm{Ca} / \mathrm{B}$ & 0.36 & $<0.003$ & $\leq 0.01$ & 0.028 & $<0.00004$ & $\leq 0.01$ & 0.077 & $<0.00004$ & $\leq 0.01$ \\
\hline $\mathrm{Ca} / \mathrm{Ba}$ & 0.69 & $=0.15$ & $>0.05$ & 0.034 & $<0.00002$ & $\leq 0.01$ & 0.050 & $<0.00007$ & $\leq 0.01$ \\
\hline $\mathrm{Ca} / \mathrm{Mn}$ & 0.87 & $=0.47$ & $>0.05$ & 0.088 & $<0.00001$ & $\leq 0.01$ & 0.101 & $<0.00001$ & $\leq 0.01$ \\
\hline $\mathrm{Mg} / \mathrm{Al}$ & 1.63 & $=\mathbf{0 . 0 3 9}$ & $>0.05$ & 0.077 & $<0.00015$ & $\leq 0.01$ & 0.047 & $<0.00001$ & $\leq 0.01$ \\
\hline $\mathrm{Mg} / \mathrm{B}$ & 0.58 & $=\mathbf{0 . 0 2 1}$ & $\leq 0.05$ & 0.044 & $<0.00017$ & $\leq 0.01$ & 0.076 & $<0.00001$ & $\leq 0.01$ \\
\hline $\mathrm{Mg} / \mathrm{Ba}$ & 1.16 & $=0.57$ & $>0.05$ & 0.062 & $<0.00012$ & $\leq 0.01$ & 0.054 & $<0.00019$ & $\leq 0.01$ \\
\hline $\mathrm{Mg} / \mathrm{Mn}$ & 1.39 & $=\mathbf{0 . 0 3 7}$ & $>0.05$ & 0.138 & $<0.00001$ & $\leq 0.01$ & 0.099 & $<0.00001$ & $\leq 0.01$ \\
\hline $\mathrm{S} / \mathrm{Al}$ & 1.35 & $=0.15$ & $>0.05$ & 0.116 & $<0.00023$ & $\leq 0.01$ & 0.086 & $<0.00001$ & $\leq 0.01$ \\
\hline $\mathrm{S} / \mathrm{B}$ & 0.45 & $<0.003$ & $\leq 0.01$ & 0.061 & $<0.00029$ & $\leq 0.01$ & 0.135 & $<0.00001$ & $\leq 0.01$ \\
\hline $\mathrm{S} / \mathrm{Ba}$ & 0.98 & $=0.93$ & $>0.05$ & 0.088 & $<0.00026$ & $\leq 0.01$ & 0.090 & $<0.00021$ & $\leq 0.01$ \\
\hline S/Mn & 1.11 & $=0.30$ & $>0.05$ & 0.211 & $<0.00001$ & $\leq 0.01$ & 0.191 & $<0.00001$ & $\leq 0.01$ \\
\hline $\mathrm{Zn} / \mathrm{Al}$ & 1.43 & $=0.21$ & $>0.05$ & 0.028 & $<0.00023$ & $\leq 0.01$ & 0.020 & $<0.00037$ & $\leq 0.01$ \\
\hline $\mathrm{Zn} / \mathrm{B}$ & 0.69 & $=0.39$ & $>0.05$ & 0.015 & $<0.013$ & $\leq 0.01$ & 0.021 & $<0.0022$ & $\leq 0.01$ \\
\hline $\mathrm{Zn} / \mathrm{Ba}$ & 1.37 & $=0.20$ & $>0.05$ & 0.029 & $<0.00006$ & $\leq 0.01$ & 0.021 & $<0.00006$ & $\leq 0.01$ \\
\hline $\mathrm{Zn} / \mathrm{Mn}$ & 1.44 & $=0.18$ & $>0.05$ & 0.049 & $<0.00006$ & $\leq 0.01$ & 0.034 & $<0.00077$ & $\leq 0.01$ \\
\hline
\end{tabular}

Table 3. Parameters of the importance (sensitivity, specificity and accuracy) of $\mathrm{Ca} / \mathrm{Al}, \mathrm{Ca} / \mathrm{B}, \mathrm{Ca} / \mathrm{Ba}, \mathrm{Ca} / \mathrm{Mn}, \mathrm{Mg} / \mathrm{Al}, \mathrm{Mg} / \mathrm{B}, \mathrm{Mg} / \mathrm{Ba}, \mathrm{Mg} / \mathrm{Mn}, \mathrm{S} / \mathrm{Al}, \mathrm{S} / \mathrm{B}, \mathrm{S} / \mathrm{Ba}, \mathrm{S} / \mathrm{Mn}, \mathrm{Zn} / \mathrm{Al}, \mathrm{Zn} / \mathrm{B}, \mathrm{Zn} / \mathrm{Ba}$, and $\mathrm{Zn} / \mathrm{Mn}$ mass fraction ratios for the diagnosis of PCa (an estimation is made for "PCa or normal and BPH prostate"). M-arithmetic mean, SD-standard deviation.

\begin{tabular}{|c|c|c|c|c|}
\hline $\begin{array}{l}\text { Mass fraction ratio } \\
\text { or their multiplication }\end{array}$ & Upper limit for PCa & Sensitivity \% & Specificity \% & Accuracy \% \\
\hline $\mathrm{Ca} / \mathrm{Al}$ & 17 & $91 \pm 9$ & $97 \pm 3$ & $96 \pm 3$ \\
\hline $\mathrm{Ca} / \mathrm{B}$ & 300 & $90 \pm 10$ & $91 \pm 5$ & $90 \pm 5$ \\
\hline $\mathrm{Ca} / \mathrm{Ba}$ & 450 & $100-9$ & $100-3$ & $100-2$ \\
\hline $\mathrm{Ca} / \mathrm{Mn}$ & 780 & $100-9$ & $100-3$ & $100-2$ \\
\hline $\mathrm{Mg} / \mathrm{Al}$ & 8.8 & $100-10$ & $100-3$ & $100-2$ \\
\hline $\mathrm{Mg} / \mathrm{B}$ & 200 & $89 \pm 11$ & $86 \pm 6$ & $89 \pm 5$ \\
\hline $\mathrm{Mg} / \mathrm{Ba}$ & 280 & $100-10$ & $97 \pm 3$ & $98 \pm 2$ \\
\hline $\mathrm{Mg} / \mathrm{Mn}$ & 370 & $90 \pm 10$ & $100-3$ & $100-2$ \\
\hline $\mathrm{S} / \mathrm{Al}$ & 100 & $91 \pm 9$ & $100-3$ & $98 \pm 2$ \\
\hline $\mathrm{S} / \mathrm{B}$ & 2500 & $89 \pm 11$ & $100-3$ & $98 \pm 2$ \\
\hline $\mathrm{S} / \mathrm{Ba}$ & 2950 & $91 \pm 9$ & $97 \pm 3$ & $96 \pm 3$ \\
\hline $\mathrm{S} / \mathrm{Mn}$ & 3200 & $91 \pm 9$ & $100-3$ & $98 \pm 2$ \\
\hline $\mathrm{Zn} / \mathrm{Al}$ & 4.6 & $100-9$ & $97 \pm 3$ & $98 \pm 2$ \\
\hline $\mathrm{Zn} / \mathrm{B}$ & 220 & $100-10$ & $97 \pm 3$ & $98 \pm 2$ \\
\hline $\mathrm{Zn} / \mathrm{Ba}$ & 150 & $100-9$ & $97 \pm 3$ & $98 \pm 2$ \\
\hline $\mathrm{Zn} / \mathrm{Mn}$ & 170 & $91 \pm 9$ & $100-3$ & $98 \pm 2$ \\
\hline$(\mathrm{Ca} / \mathrm{Ba}) \cdot(\mathrm{Mg} / \mathrm{Al}) \cdot(\mathrm{Zn} / \mathrm{Mn})$ & 100000 & $100-9$ & $100-3$ & $100-2$ \\
\hline$(\mathrm{Ca} / \mathrm{Ba}) \cdot(\mathrm{Mg} / \mathrm{Al}) \cdot(\mathrm{S} / \mathrm{B}) \cdot(\mathrm{Zn} / \mathrm{Mn})$ & 130000 & $100-9$ & $100-3$ & $100-2$ \\
\hline
\end{tabular}

$\mathrm{Ca} / \mathrm{Ba}, \mathrm{Ca} / \mathrm{Mn}, \mathrm{Mg} / \mathrm{Al}, \mathrm{Mg} / \mathrm{B}, \mathrm{Mg} / \mathrm{Ba}, \mathrm{Mg} / \mathrm{Mn}, \mathrm{S} / \mathrm{Al}, \mathrm{S} / \mathrm{B}, \mathrm{S} / \mathrm{Ba}, \mathrm{S} /$ $\mathrm{Mn}, \mathrm{Zn} / \mathrm{Al}, \mathrm{Zn} / \mathrm{B}, \mathrm{Zn} / \mathrm{Ba}$, and $\mathrm{Zn} / \mathrm{Mn}$ mass fraction ratios as well as for $(\mathrm{Ca} / \mathrm{Ba}) \cdot(\mathrm{Mg} / \mathrm{Al}) \cdot(\mathrm{Zn} / \mathrm{Mn})$ and $(\mathrm{Ca} / \mathrm{Ba}) \cdot(\mathrm{Mg} / \mathrm{Al}) \cdot(\mathrm{S} / \mathrm{B}) \cdot(\mathrm{Zn} / \mathrm{Mn})$ multiplications of mass fraction ratios in all samples of normal, benign hypertrophic and cancerous prostate, respectively.

\section{Discussion}

As was shown by us $[18,22,47]$ the use of CRM IAEA H-4, INCTSBF-4 Soya Bean Flour, INCT-TL-1 Tea Leaves, and INCT-MPH-2 Mixed Polish Herbs as certified reference materials for the analysis of samples of prostate tissue can be seen as quite acceptable. Good agreement of the $\mathrm{Al}, \mathrm{B}, \mathrm{Ba}, \mathrm{Ca}, \mathrm{Mg}, \mathrm{Mn}, \mathrm{S}$, and $\mathrm{Zn}$ contents analyzed by INAA-SLR and ICP-AES with the certified data of reference materials indicated an acceptable accuracy of the results obtained in the study and presented in Tables land 2.

The mean values and all selected statistical parameters were calculated for sixteen mass fraction ratios: $\mathrm{Ca} / \mathrm{Al}, \mathrm{Ca} / \mathrm{B}, \mathrm{Ca} / \mathrm{Ba}, \mathrm{Ca} / \mathrm{Mn}$,

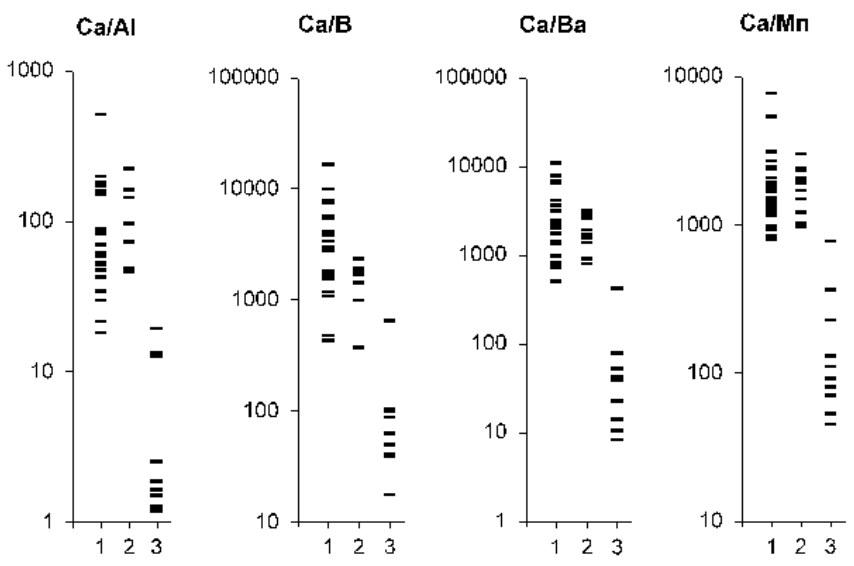

Figure 1. Individual data sets for $\mathrm{Ca} / \mathrm{Al}, \mathrm{Ca} / \mathrm{B}, \mathrm{Ca} / \mathrm{Ba}$, and $\mathrm{Ca} / \mathrm{Mn}$ mass fraction ratios in samples of normal (1) benign hypertrophic (2) and cancerous (3) prostate. 


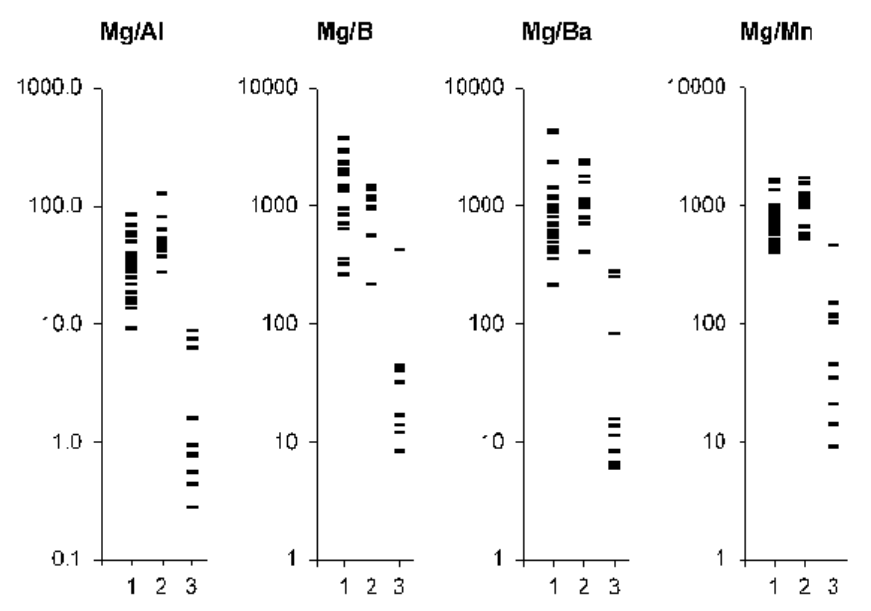

Figure 2. Individual data sets for $\mathrm{Mg} / \mathrm{Al}, \mathrm{Mg} / \mathrm{B}, \mathrm{Mg} / \mathrm{Ba}$, and $\mathrm{Mg} / \mathrm{Mn}$ mass fraction ratios in samples of normal (1) benign hypertrophic (2) and cancerous (3) prostate.

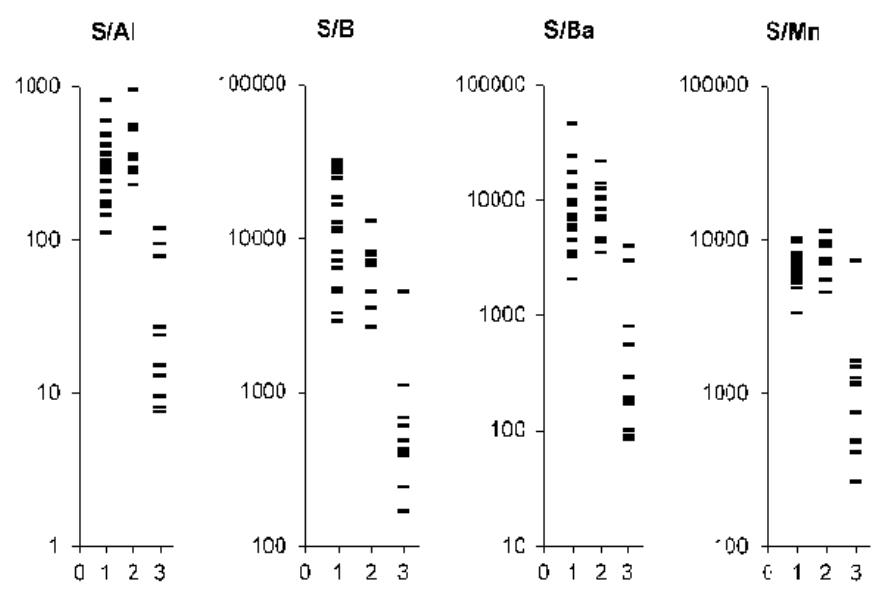

Figure 3. Individual data sets for $\mathrm{S} / \mathrm{Al}, \mathrm{S} / \mathrm{B}, \mathrm{S} / \mathrm{Ba}$, and $\mathrm{S} / \mathrm{Mn}$ mass fraction ratios in samples of normal (1) benign hypertrophic (2) and cancerous (3) prostate.
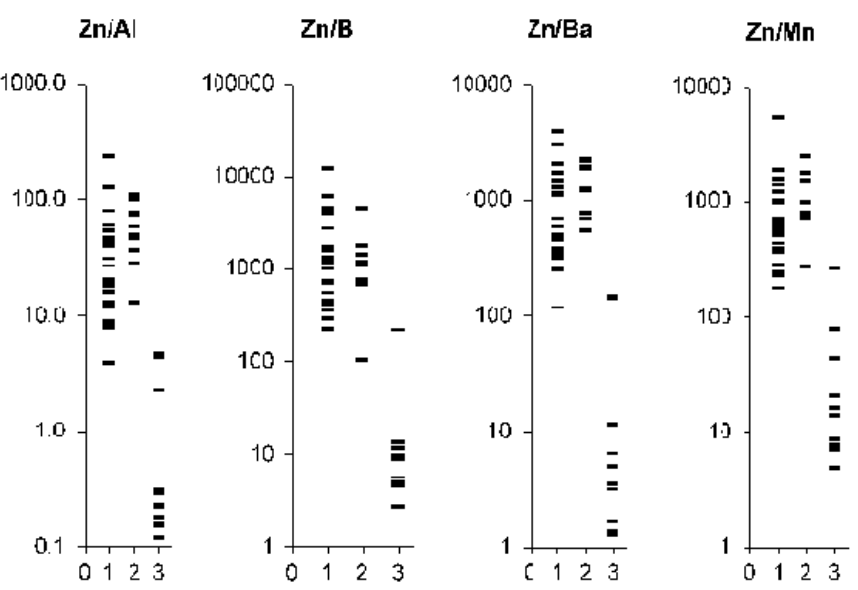

Figure 4. Individual data sets for $\mathrm{Zn} / \mathrm{Al}, \mathrm{Zn} / \mathrm{B}, \mathrm{Zn} / \mathrm{Ba}$, and $\mathrm{Zn} / \mathrm{Mn}$ mass fraction ratios in samples of normal (1) benign hypertrophic (2) and cancerous (3) prostate.

$\mathrm{Mg} / \mathrm{Al}, \mathrm{Mg} / \mathrm{B}, \mathrm{Mg} / \mathrm{Ba}, \mathrm{Mg} / \mathrm{Mn}, \mathrm{S} / \mathrm{Al}, \mathrm{S} / \mathrm{B}, \mathrm{S} / \mathrm{Ba}, \mathrm{S} / \mathrm{Mn}, \mathrm{Zn} / \mathrm{Al}, \mathrm{Zn} / \mathrm{B}, \mathrm{Zn} /$ $\mathrm{Ba}$, and $\mathrm{Zn} / \mathrm{Mn}$ (Table 1). The mass fraction of these chemical elements were measured in all, or a major portion of normal prostate samples. The masses of $\mathrm{BPH}$ and PCa samples varied very strong from a few
(CaiBa) (Mg/Al) (Zn/Mn)

$(\mathrm{Ca} / \mathrm{Ba})(\mathrm{Mg} / \mathrm{Al})(\mathrm{S} / \mathrm{B})(\mathrm{Zn} / \mathrm{Mn})$
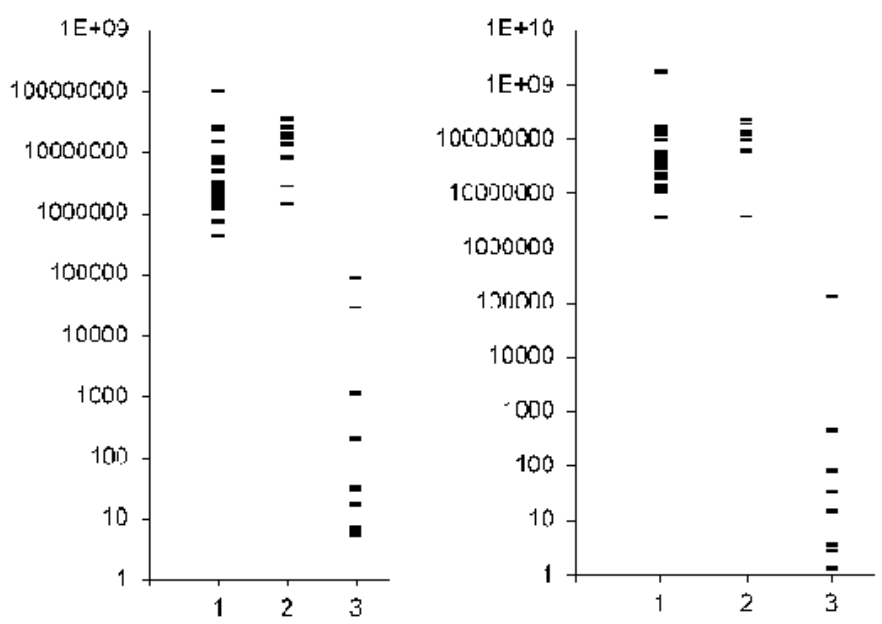

Figure 5. Individual data sets for $(\mathrm{Ca} / \mathrm{Ba}) \cdot(\mathrm{Mg} / \mathrm{Al}) \cdot(\mathrm{Zn} / \mathrm{Mn})$ and $(\mathrm{Ca} / \mathrm{Ba}) \cdot(\mathrm{Mg} / \mathrm{Al}) \cdot(\mathrm{S} /$ $\mathrm{B}) \cdot(\mathrm{Zn} / \mathrm{Mn})$ test in samples of normal (1) benign hypertrophic (2) and cancerous (3) prostate.

milligrams (sample from needle biopsy material) to $100 \mathrm{mg}$ (sample from resected material). Therefore, in BPH and PCa prostates mass fractions of $\mathrm{Zn}$ were measured in all samples, while mass fractions of other chemical elements were determined in 22 samples (11 BPH and $11 \mathrm{PCa}$ samples, respectively). Thus, the $\mathrm{Ca} / \mathrm{Al}, \mathrm{Ca} / \mathrm{B}, \mathrm{Ca} / \mathrm{Ba}, \mathrm{Ca} / \mathrm{Mn}$, $\mathrm{Mg} / \mathrm{Al}, \mathrm{Mg} / \mathrm{B}, \mathrm{Mg} / \mathrm{Ba}, \mathrm{Mg} / \mathrm{Mn}, \mathrm{S} / \mathrm{Al}, \mathrm{S} / \mathrm{B}, \mathrm{S} / \mathrm{Ba}, \mathrm{S} / \mathrm{Mn}, \mathrm{Zn} / \mathrm{Al}, \mathrm{Zn} / \mathrm{B}, \mathrm{Zn} /$ $\mathrm{Ba}$, and $\mathrm{Zn} / \mathrm{Mn}$ mass fraction ratios were calculated in all, or a major portion of normal prostates, and only in 22 hyperplastic and cancerous prostates.

The results presented in Table 1 showed substantial differences between the arithmetic means and the medians, which impeached a normal distribution of the investigated elemental content ratios. These findings were a motive for us to compare the means of mass fraction ratios in normal, BPH and PCa prostate using parametric Student's $t$-test and nonparametric Wilcoxon-Mann-Whitney $U$-test. No published data referring to mass fraction ratios of chemical elements in the human prostate was found.

From Table 2, it is observed that in benign hypertrophic tissues the $\mathrm{Ca} / \mathrm{Al}, \mathrm{Ca} / \mathrm{Ba}, \mathrm{Ca} / \mathrm{Mn}, \mathrm{Mg} / \mathrm{Ba}, \mathrm{S} / \mathrm{Al}, \mathrm{S} / \mathrm{Ba}, \mathrm{S} / \mathrm{Mn}, \mathrm{Zn} / \mathrm{Al}, \mathrm{Zn} / \mathrm{B}, \mathrm{Zn} /$ $\mathrm{Ba}$, and $\mathrm{Zn} / \mathrm{Mn}$ mass fraction ratios do not differ from normal levels while the $\mathrm{Mg} / \mathrm{Al}$ and $\mathrm{Mg} / \mathrm{Mn}$ ratios are higher and $\mathrm{Ca} / \mathrm{B}, \mathrm{Mg} / \mathrm{B}$, and $\mathrm{S} / \mathrm{B}$ ratios are lower. In cancerous tissue all ratios of chemical element mass fractions investigated were significantly lower than in normal parenchyma of the prostate. All these mass fraction ratios show similar variations in cancerous tissues when compared with benign hypertrophic tissues of the prostate.

Analysis of elemental content ratios in prostate tissue could become a powerful diagnostic tool. To a large extent, the resumption of the search for new methods for early diagnosis of PCa was due to experience gained in a critical assessment of the limited capacity of the prostate specific antigen (PSA) serum test $[81,82]$. In addition to the PSA serum test and morphological study of needle-biopsy cores of the prostate, the development of other highly precise testing methods seems to be very useful. Experimental conditions of the present study were approximated to the hospital conditions as closely as possible. In all cases we analyzed a part of the material obtained from a biopsy of the indurated site in the prostate. Therefore, our data allow us to 
evaluate adequately the importance of elemental content ratios for the diagnosis of PCa. As is evident from individual data sets (Figures 1-4) and Table 3 , the values of all mass fraction ratios are very informative for a differential diagnosis. For example, if 450 is the value of $\mathrm{Ca} / \mathrm{Ba}$ mass fraction ratio assumed to be the upper limit for $\mathrm{PCa}$ (Figure 1 and Table 3) and an estimation is made for "PCa or intact and BPH tissue", the following values was obtained:

Sensitivity $=\{$ True Positives $(\mathrm{TP}) /[\mathrm{TP}+$ False Negatives $(\mathrm{FN})]\} \cdot 100 \%=(100-9) \%$;

Specificity $=\{$ True Negatives $(\mathrm{TN}) /[\mathrm{TN}+$ False Positives (FP) $]\} \cdot 100 \%=(100-3) \%$;

$$
\text { Accuracy }=[(\mathrm{TP}+\mathrm{TN}) /(\mathrm{TP}+\mathrm{FP}+\mathrm{TN}+\mathrm{FN})] \cdot 100 \%=(100-2) \% .
$$

The number of prostates investigated was taken into account for calculation of confidence intervals [83]. In other words, if $\mathrm{Ca} / \mathrm{Ba}$ mass fraction ratio in a prostate biopsy sample does not higher 450 , one could diagnose a malignant tumor with an accuracy of (100-2)\%. Thus, using the $(\mathrm{Ca} / \mathrm{Ba})$-test makes it possible to diagnose cancer in (100-9)\% cases (sensitivity).

It should be noted, that all ratios of chemical element mass fraction investigated in the study are very informative for the diagnosis of PCa and these tests have good levels of sensitivity, specificity and accuracy varied in ranges $89 \%-100 \%, 86 \%-100 \%$, and $89 \%-100 \%$, respectively (Table 3). However, it is possible to increase a separation distance between the value of "Upper limit" for PCa and the lowest values among normal and BPH results if use a combination of the selected ratios. For example, for this purpose a multiplication of $(\mathrm{Ca} / \mathrm{Ba}) \cdot(\mathrm{Mg} / \mathrm{Al}) \cdot(\mathrm{Zn} /$ $\mathrm{Mn})$ or $(\mathrm{Ca} / \mathrm{Ba}) \cdot(\mathrm{Mg} / \mathrm{Al}) \cdot(\mathrm{S} / \mathrm{B}) \cdot(\mathrm{Zn} / \mathrm{Mn})$ can be used. If the level 130000 was accepted as the "Upper limit" of $(\mathrm{Ca} / \mathrm{Ba}) \cdot(\mathrm{Mg} / \mathrm{Al}) \cdot(\mathrm{S} / \mathrm{B}) \cdot(\mathrm{Zn} / \mathrm{Mn})$ test for the diagnosis of $\mathrm{PCa}$ (Figure 5), the sensitivity, specificity and accuracy of this test are $100-9 \%, 100-3 \%$, and $100-2 \%$, respectively and the lowest value in normal and $\mathrm{BPH}$ prostate is almost 30 time higher the highest value in cancerous prostate.

Mass fraction ratios of $\mathrm{Ca} / \mathrm{Al}, \mathrm{Ca} / \mathrm{Ba}, \mathrm{Ca} / \mathrm{Mn}, \mathrm{Mg} / \mathrm{Ba}, \mathrm{S} / \mathrm{Al}, \mathrm{S} / \mathrm{Ba}$, $\mathrm{S} / \mathrm{Mn}, \mathrm{Zn} / \mathrm{Al}, \mathrm{Zn} / \mathrm{B}, \mathrm{Zn} / \mathrm{Ba}$, and $\mathrm{Zn} / \mathrm{Mn}$ in the needle-biopsy cores could be used as a tool to diagnose PCa and are comparable with characteristics of the $\mathrm{Zn}$ mass fraction-test $[67,68]$. However, it is our opinion that application of the elemental content ratios is more suitable for PCa diagnosis. Elemental mass fraction depends on the sample mass, which decreases with loss of its moisture. The needle-biopsy core is a small piece of tissue with a relatively high "surface/volume" ratio. After sampling, it begins to lose mass very fast. Weight loss of samples depends on the humidity of operating and store rooms [69]. Thus, it is very difficult to determine the fresh mass of needle-biopsy cores and to calculate the precise mass fraction of chemical elements. Sample freeze-dry, storage in air-tight vials until weighing, and then calculating mass fraction on dry mass basis is the only possible method that eliminates the variation in sample mass. Conversely, accuracy of elemental content ratios does not depend on sample mass and changes in moisture content. Therefore, this method does not require dry samples. Moreover, the use of the relations between mass fractions of chemical elements is particularly promising for the development of in vivo diagnostic methods, including the diagnosis of PCa.

\section{Conclusion}

The combination of nondestructive INAA-SLR and destructive ICP-AES methods is satisfactory analytical tool for the precise determination of chemical element mass fractions and their ratios in the tissue samples of normal, $\mathrm{BPH}$ and carcinomatous prostate glands. In this work, mean values of $\mathrm{Ca} / \mathrm{Al}, \mathrm{Ca} / \mathrm{Ba}, \mathrm{Ca} / \mathrm{Mn}, \mathrm{Mg} / \mathrm{Ba}, \mathrm{S} / \mathrm{Al}, \mathrm{S} /$ $\mathrm{Ba}, \mathrm{S} / \mathrm{Mn}, \mathrm{Zn} / \mathrm{Al}, \mathrm{Zn} / \mathrm{B}, \mathrm{Zn} / \mathrm{Ba}$, and $\mathrm{Zn} / \mathrm{Mn}$ mass fraction ratios in normal, benign hyperplastic and cancerous prostate were calculated using data of INAA-SLR and ICP-AES. It was observed that in benign hyperplastic prostate the $\mathrm{Ca} / \mathrm{Al}, \mathrm{Ca} / \mathrm{Ba}, \mathrm{Ca} / \mathrm{Mn}, \mathrm{Mg} / \mathrm{Ba}, \mathrm{S} / \mathrm{Al}, \mathrm{S} / \mathrm{Ba}, \mathrm{S} /$ $\mathrm{Mn}, \mathrm{Zn} / \mathrm{Al}, \mathrm{Zn} / \mathrm{B}, \mathrm{Zn} / \mathrm{Ba}$, and $\mathrm{Zn} / \mathrm{Mn}$ mass fraction ratios do not differ from normal levels while the $\mathrm{Mg} / \mathrm{Al}$ and $\mathrm{Mg} / \mathrm{Mn}$ ratios are higher and $\mathrm{Ca} / \mathrm{B}, \mathrm{Mg} / \mathrm{B}$, and $\mathrm{S} / \mathrm{B}$ ratios are lower. In cancerous prostate the $\mathrm{Ca} /$ $\mathrm{Al}, \mathrm{Ca} / \mathrm{Ba}, \mathrm{Ca} / \mathrm{Mn}, \mathrm{Mg} / \mathrm{Ba}, \mathrm{S} / \mathrm{Al}, \mathrm{S} / \mathrm{Ba}, \mathrm{S} / \mathrm{Mn}, \mathrm{Zn} / \mathrm{Al}, \mathrm{Zn} / \mathrm{B}, \mathrm{Zn} / \mathrm{Ba}$, and $\mathrm{Zn} / \mathrm{Mn}$ mass fraction ratios are significantly lower than in normal and benign hyperplastic prostate. It was shown that all ratios of chemical element mass fractions investigated in the study are informative for the prostate cancer diagnosis. Finally, we propose to use the estimation of selected elemental mass fraction ratios and some theirs multiplications in a needle-biopsy core as an accurate tool to diagnose prostate cancer. Further studies on larger number of samples are required to confirm our findings, to study the impact of the elemental mass fraction ratios in gland tissue on prostate cancer etiology and to examine the longterm pathological outcome.

\section{Acknowledgements}

We are grateful to Dr. Tatyana Sviridova, Medical Radiological Research Center, Obninsk, and to the late Prof. A.A. Zhavoronkov, Institute of Human Morphology, Russian Academy of Medical Sciences, Moscow, for supplying prostate samples. We are also grateful to Dr. Karandaschev V., Dr. Nosenko S., and Moskvina I., Institute of Microelectronics Technology and High Purity Materials, Chernogolovka, Russia, for their help in ICP-AES analysis.

\section{References}

1. Kirby RS (2000) The natural history of benign prostatic hyperplasia: what have we learned in the last decade. Urology 56: 3-6. [Crossref]

2. Roehrborn C, McConnell J (2002) Etiology, pathophysiology, epidemiology and natural history of benign prostatic hyperplasia. In: Walsh P, Retik A, Vaughan E, Wein A (Eds) Campbell's Urology, 8th Edn, Philadelphia: Saunders, p: 1297-1336.

3. Lepor H (2005) Pathophysiology of benign prostatic hyperplasia in the aging male population. Rev Urol 7 Suppl 4: S3-3S12. [Crossref]

4. Oliver SE, Gunnell D, Donovan JL (2000) Comparison of trends in prostate-cancer mortality in England and Wales and the USA. Lancet 355: 1788-1789. [Crossref]

5. Kumar RJ, Barqawi AB, Crawford ED (2004) Epidemiology of prostate cancer Business Briefing: US Oncology Review: 1-6.

6. Maddams J, Brewster D, Gavin A, Steward J, Elliott J, et al. (2009) Cancer prevalence in the United Kingdom: estimates for 2008. Br J Cancer 101: 541-547. [Crossref]

7. Lutz JM, Francisci S, Mugno E, Usel M, Pompe-Kirn V, et al. (2003) Cancer prevalence in Central Europe: the EUROPREVAL Study. Ann Oncol 14: 313-322. [Crossref]

8. Möller T, Anderson H, Aareleid T, Hakulinen T, Storm H, et al. (2003) Cancer prevalence in Northern Europe: the EUROPREVAL study. Ann Oncol 14: 946-957. [Crossref]

9. De Angelis R, Grande E, Inghelmann R, Francisci S, Micheli A, et al. (2007) Cancer prevalence estimates in Italy from 1970 to 2010. Tumori 93: 392-397. [Crossref]

10. Waalkes MP, Rehm S (1994) Cadmium and prostate cancer. J Toxicol Environ Health 43: 251-269. [Crossref]

11. Zaichick V, Zaichick S (1999) Role of zinc in prostate cancerogenesis. In: Anke M (Ed) Jena: Friedrich-Schiller-Universitat p: 104-115.

12. Platz EA, Helzlsouer KJ (2001) Selenium, zinc, and prostate cancer. Epidemiol Rev 23: 93-101. [Crossref]

13. Zaichick V (2004) INAA and EDXRF applications in the age dynamics assessment of $\mathrm{Zn}$ content and distribution in the normal human prostate. J Radioanal Nucl Chem 262: $229-234$ 
14. Gray MA, Centeno JA, Slaney DP, Ejnik JW, Todorov T, et al. (2005) Environmental exposure to trace elements and prostate cancer in three New Zealand ethnic groups. Int J Environ Res Public Health 2: 374-384. [Crossref]

15. Zaichick S, Zaichick V (2011) INAA application in the age dynamics assessment of $\mathrm{Br}$ $\mathrm{Ca}, \mathrm{Cl}, \mathrm{K}, \mathrm{Mg}, \mathrm{Mn}$, and $\mathrm{Na}$ content in the normal human prostate. $\mathrm{J}$ Radioanal Nucl Chem 288: 197-202.

16. Zaichick S, Zaichick V (2011) The effect of age on Ag, Co, Cr, Fe, Hg, Sb, Sc, Se, and $\mathrm{Zn}$ contents in intact human prostate investigated by neutron activation analysis. Appl Radiat Isot 69: 827-833.

17. Zaichick S, Zaichick V (2011) The Br, Fe, Rb, Sr, and Zn content and interrelation in intact and morphologic normal prostate tissue of adult men investigated by energy dispersive X-ray fluorescent analysis. X-Ray Spectrom 40: 464-469.

18. Zaichick V, Nosenko S, Moskvina I (2012) The effect of age on 12 chemical element contents in the intact prostate of adult men investigated by inductively coupled plasma atomic emission spectrometry. Biol Trace Elem Res 147: 49-58. [Crossref]

19. Zaichick S, Zaichick V, Nosenko S, Moskvina I (2012) Mass Fractions of 52 Trace Elements and Zinc Trace Element Content Ratios in Intact Human Prostates Investigated by Inductively Coupled Plasma Mass Spectrometry. Biol Trace Elem Res 149: 171-183.

20. Zaichick V, Zaichick S (2014) Age-related histological and zinc content changes in adult nonhyperplastic prostate glands. Age (Dordr) 36: 167-181. [Crossref]

21. Zaichick V, Zaichick S2 (2014) INAA application in the assessment of chemical element mass fractions in adult and geriatric prostate glands. Appl Radiat Isot 90: 6273. [Crossref]

22. Zaichick V, Zaichick S (2014) Determination of trace elements in adults and geriatric prostate combining neutron activation with inductively coupled plasma atomic emission spectrometry. Open Journal of Biochemistry 1: 16-33.

23. Zaichick V, Zaichick S (2014) Use of INAA and ICP-MS for the assessment of trace element mass fractions in adult and geriatric prostate. J Radioanal Nucl Chem 301: 383-397.

24. Zaichick V (2015) The Variation with Age of 67 Macro- and Microelement Contents in Nonhyperplastic Prostate Glands of Adult and Elderly Males Investigated by Nuclear Analytical and Related Methods. Biol Trace Elem Res 168: 44-60. [Crossref]

25. Zaichick V, Zaichick S (2015) Dietary intake of minerals and prostate cancer: insights into problem based on the chemical element contents in the prostate gland. J Aging Res Clin Practice 4: 164-171.

26. Zaichick V, Zaichick S (2015) Global contamination from uranium: insights into problem based on the uranium content in the human prostate gland. $J$ Environ Health Sci 1: 1-5.

27. Zaichick V, Zaichick S (2016) Variations in concentration and distribution of several androgen-dependent and -independent trace elements in nonhyperplastic prostate gland tissue throughout adulthood. J Androl Gynaecol 4: 1-10.

28. Zaichick V, Zaichick S (2016) Age-related changes in concentration and histological distribution of $\mathrm{Br}, \mathrm{Ca}, \mathrm{Cl}, \mathrm{K}, \mathrm{Mg}, \mathrm{Mn}$, and $\mathrm{Na}$ in nonhyperplastic prostate of adults. European Journal of Biology and Medical Science Research 4: 31-48.

29. Zaichick V, Zaichick S (2016) Variations in concentration and histological distribution of $\mathrm{Ag}, \mathrm{Co}, \mathrm{Cr}, \mathrm{Fe}, \mathrm{Hg}, \mathrm{Rb}, \mathrm{Sb}, \mathrm{Sc}, \mathrm{Se}$, and $\mathrm{Zn}$ in nonhyperplastic prostate gland throughout adulthood. J Androl Gynaecol 2: 011.

30. Zaichick V (2006) Medical elementology as a new scientific discipline. $J$ Radioanal Nucl Chem 269: 303-309.

31. Tipton IH, Cook MJ (1963) Trace elements in human tissue. II. Adult subjects from the United States. Health Phys 9: 103-145. [Crossref]

32. Stitch SR (1957) Trace elements in human tissue.1. A semi-quantitative spectrographic survey. Biochem J 67: 97-103. [Crossref]

33. Tipton JH, Steiner RL, Foland WD, Mueller J, Stanley M (1954) USAEC-ORNLReport-CF-54: 12-66.

34. Jaritz M, Anke M, Holzinger S (1998) Der Bariumgehalt verschiedener Organe von Feldhase, Wildschwein, Damhirsch, Reh, Rothirsch, Mufflon and Mensch. In: Anke M (Ed) Mengen und Spurenelemente p: 467-474.

35. Kubo H, Hashimoto S, Ishibashi A, Chiba R, Yokota H (1976) Simultaneous determinations of $\mathrm{Fe}, \mathrm{Cu}, \mathrm{Zn}$, and $\mathrm{Br}$ concentrations in human tissue sections. Medical Physics 3: 204-209.

36. Zaichick S, Zaichick V (2010) Method and portable facility for energy-dispersive X-ray fluorescent analysis of zinc content in needle-biopsy specimens of prostate. X-Ray Spectrom 39: 83-89.

37. Schneider HJ, Anke M, Holm W (1970) The inorganic components of testicle, epididymis, seminal vesicle, prostate and ejaculate of young men. Int Urol Nephrol 2: 419-427.

38. Tohno S, Kobayashi M, Shimizu H, Tohno Y, Suwannahoy P, et al. (2009) Age-related changes of the concentrations of select elements in the prostates of Japanese. Biol Trace Elem Res 127: 211-227. [Crossref]

39. Anspaugh LR, Robinson WL, Martin WH, Lowe OA (1973) Compilation of Published Information on Elemental Concentrations in human Organs in Both Normal and Diseased States. NTRL p: 1-4.

40. Jafa A, Mahendra NM, Chowdhury AR, Kamboj VP (1980) Trace elements in prostatic tissue and plasma in prostatic diseases of man. Indian J Cancer 17: 34-37. [Crossref]

41. Sangen H (1967) The influence of the trace metal on aconitase activity in human prostate glands. Nihon Hinyokika Gakkai Zasshi 58: 1146-1159. [Crossref]

42. Guntupalli JNR, Padala S, Gummuluri AVRM, Muktineni RK, Byreddy SR, et al (2007) Trace elemental analysis of normal, benign hypertrophic and cancerous tissues of the prostate gland using the particle-induced X-ray emission technique. Eur $J$ Cancer Prev 16: 108-115.

43. Zaichick V, Zaichick S (2013) The effect of age on $\mathrm{Br}, \mathrm{Ca}, \mathrm{Cl}, \mathrm{K}, \mathrm{Mg}, \mathrm{Mn}$, and $\mathrm{Na}$ mas fraction in pediatric and young adult prostate glands investigated by neutron activation analysis. Appl Radiat Isot 82: 145-151. [Crossref]

44. Forssén A (1972) Inorganic elements in the human body. I. Occurrence of Ba, Br, Ca, $\mathrm{Cd}, \mathrm{Cs}, \mathrm{Cu}, \mathrm{K}, \mathrm{Mn}, \mathrm{Ni}, \mathrm{Sn}, \mathrm{Sr}, \mathrm{Y}$ and $\mathrm{Zn}$ in the human body. Ann Med Exp Biol Fenn 50: 99-162. [Crossref]

45. Banas A, Kwiatek WM, Zajac W (2001) Trace element analysis of tissue section by means of synchrotron radiation: the use of GNUPLOT for SPIXE spectra analysis Journal of Alloys and Compounds 328: 135-138.

46. Soman SD, Joseph KT, Raut SJ, Mulay CD, Parameshwaran M, et al. (1970) Studies on major and trace element content in human tissues. Health Phys 19: 641-656. [Crossref]

47. Zaichick V, Zaichick S (2013) NAA-SLR and ICP-AES application in the assessmen of mass fraction of 19 chemical elements in pediatric and young adult prostate glands. Biol Trace Elem Res 156: 357-366. [Crossref]

48. Belt TH, Irwin D, King EJ (1936) Silicon and dust deposits in the tissues of persons without occupational exposure to siliceous dusts. Can Med Assoc J 34: 125-133. [Crossref]

49. Galván-Bobadilla AI, García-Escamilla RM, Gutiérrez-García N, Mendoza-Magaña ML, Rosiles-Martínez R (2005) Cadmium and zinc concentrations in prostate cancer and benign prostate hyperplasia. Rev Latinoamer Patol Clin 52: 109-117.

50. Zaichick S, Zaichick V (2014) EDXRF determination of trace element contents in benign prostatic hypertrophic tissue. In: Fundamental Interactions and Neutrons, Neutron Spectroscopy, Nuclear Structure, Ultracold Neutrons, Related Topics. Dubna, Moscow Region, Joint Institute for Nuclear Research, Russia p: 311-316.

51. Hienzsch E, Schneider HJ, Anke M (1970) Vergleichende Untersuchungen zum Mengen- und Spurenelementgehalt der normalen Prostata, des Prostataadenoms und des Prostatakarzinoms. Zeitschrift für Urologie und Nephrologie 63: 543-546.

52. Leitão RG, Palumbo A, Souza PAVR, Pereira GR, Canellas CGL, et al. (2014) Elemental concentration analysis in prostate tissues using total reflection X-ray fluorescence. Radiation Physics and Chemistry 95: 62-64.

53. Yaman M, Atici D, Bakirdere S, Akdeniz I (2005) Comparison of trace metal concentrations in malign and benign human prostate. $J$ Med Chem 48: 630-634. [Crossref]

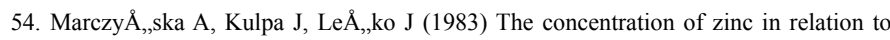
fundamental elements in the diseased human prostate. Int Urol Nephrol 15: 257-265. [Crossref]

55. Picurelli L1, Olcina PV, Roig MD, Ferrer J (1991) [Determination of Fe, Mg, Cu, and $\mathrm{Zn}$ in normal and pathological prostatic tissue]. Actas Urol Esp 15: 344-350. [Crossref]

56. Györkey F, Min KW, Huff JA, Györkey P (1967) Zinc and magnesium in human prostate gland: normal, hyperplastic, and neoplastic. Cancer Res 27: 1348-1353. [Crossref]

57. Kwiatek WM, Banas A, Banas K, Podgorczyk M, Dyduch G, et al. (2006) Distinguishing prostate cancer from hyperplasia. Acta Physica Polonica 109: 377-381.

58. Kiziler AR, Aydemir B, Guzel S, Alici B, Ataus S, et al. (2010) May the level and ratio 
changes of trace elements be utilized in identification of disease progression and grade in prostatic cancer. Trace Elements and Electrolytes 27: 65-72.

59. Eckhert CD (2005) Microlocalization and quantitation of risk associated elements in gleason graded prostate tissue. Annual Report, University of California, Los Angeles, CA p: $1-12$.

60. Paluszkiewicz C, Kwiatek W (2001) Analysis of human cancer prostate tissue using FTIR microspectroscopy and SRIXE techniques. J Mol Struct 565-566: 329-334.

61. Kwiatek WM, Banas A, Gajda M, Galka M, Pawlicki B, et al. (2005) Cancerous tissues analyzed by SRIXE. Journal of Alloys and Compounds 401: 173-177.

62. Kwiatek WM, Hanson AL, Paluszkiewicz C, Galka, Gajda M, et al. (2004) Application of SRIXE and XANES to the determination of the oxidation state of iron in prostate tissue sections. Journal of Alloys and Compounds 362: 83-87.

63. Hienzsch E, Schneider HJ, Anke M (1970) Comparative studies of the number and amount of trace elements of the normal prostate, prostate adenoma and prostate carcinoma. Z Urol Nephrol 63: 543-546. [Crossref]

64. Dhar NK, Goel TC, Dube PC, Chowdhury AR, Kar AB (1973) Distribution and concentration of zinc in the subcellular fractions of benign hyperplastic and malignant neoplastic human prostate. Exp Mol Pathol 19: 139-142.

65. Zaichick V, Zaichick S (2016) Prostatic tissue level of some major and trace elements in patients with BPH. Jacobs Journal of Nephrology and Urology 3: 025.

66. Zaichick V, Zaichick S (2016) The Bromine, Calcium, Potassium, Magnesium, Manganese, and Sodium Contents in Adenocarcinoma of Human Prostate Gland. $J$ Hematology and Oncology Research 2(2):1-12.

67. Zaichick VY, Sviridova TV, Zaichick SV (1997) Zinc in the human prostate gland: normal, hyperplastic and cancerous. Int Urol Nephrol 29: 565-574. [Crossref]

68. Zaichick S, Zaichick V (2012) Trace elements of normal, benign hypertrophic and cancerous tissues of the human prostate gland investigated by neutron activation analysis. Appl Radiat Isot 70: 81-87.

69. Zaichick V (1997) Sampling, sample storage and preparation of biomaterials for INAA in clinical medicine, occupational and environmental health. In: Harmonization of Health-Related Environmental Measurements Using Nuclear and Isotopic Techniques. Vienna: IAEA p: 123-133.

70. Zaichick V, Zaichick S (1996) Instrumental effect on the contamination of biomedica samples in the course of sampling. The Journal of Analytical Chemistry 51: 1200-1205.
71. Zaichick V, Zaichick S (1997) A search for losses of chemical elements during freezedrying of biological materials. J Radioanal Nucl Chem 218: 249-253.

72. Zaichick V (2004) Losses of chemical elements in biological samples under the dry aching process. Trace Elements in Medicine 5: 17-22.

73. Zaichick V, Zaichick S, Davydov G (2015) Differences between chemical element contents in hyperplastic and nonhyperplastic prostate glands investigated by neutron activation analysis. Biol Trace Elem Res 164: 25-35. [Crossref]

74. Zaichick S, Zaichick V (2015) Prostatic Tissue Level of some Androgen Dependen and Independent Trace Elements in Patients with Benign Prostatic Hyperplasia. Androl Gynecol: Curr Res 3: 3.

75. Zaichick V, Zaichick S (2016) Prostatic tissue level of some major and trace element in patients with BPH. JJ Nephro Urol 3: 025.

76. Zaichick V, Zaichick S (2016) The bromine, calcium, potassium, magnesium, manganese, and sodium contents in adenocarcinoma of human prostate gland. $J$ Hematology and Oncology Research 2: 1-12.

77. Zaichick V, Zaichick S (2016) Age-related changes in concentration and histologica distribution of 18 chemical elements in nonhyperplastic prostate of adults. WJPMR 2: 5-18.

78. Zaichick V, Zaichick S (2016) The Comparison between the contents and interrelationships of 17 chemical elements in normal and cancerous prostate gland. Journal of Prostate Cancer 1: 105.

79. Zaichick V, Zaichick S (2016) Chemical elemental content/Calcium ratios in tissue of human hyperplastic prostate gland. Journal of Applied Life Sciences International 4: $1-11$.

80. Korelo AM, Zaichick V (1993) Software to optimize the multielement INAA of medica and environmental samples. In: Activation Analysis in Environment Protection. Dubna, Moscow Region, Russia: Joint Institute for Nuclear Research p: 326-332.

81. Catalona WJ1 (1996) Clinical utility of measurements of free and total prostate-specific antigen (PSA): a review. Prostate Suppl 7: 64-69. [Crossref]

82. Hjertholm P, Fenger-Gron M, Vestergaard M, Christensen MB, Borre M, et al. (2015) Variation in general practice prostate-specific antigen testing and prostate cancer outcomes: An ecological study. Int J Cancer 136: 435-442.

83. Genes VS (1967) Simple methods for cybernetic data treatment of diagnostic and physiological studies. Moscow, Nauka.

Copyright: $\odot 2016$ Zaichick V. This is an open-access article distributed under the terms of the Creative Commons Attribution License, which permits unrestricted use, distribution, and reproduction in any medium, provided the original author and source are credited. 